processes involving a particular species of atom or ion. It is only to be hoped that the price does not prevent the appearance of these volumes on the shelves of all interested in atomic collisions, where surely they deserve pride of place.

B. H. Branspen

\section{COURSE IN QUANTUM MECHANICS}

\section{Lectures on Quantum Mechanics}

By Gordon Baym. (Lecture Notes and Supplements in Physics.) Pp. xi +594. (Benjamin: New York and Amsterdam, 1969.) n.p.

THe dust-jacket correctly describes this book as a supplement to a graduate course in quantum mechanies; it aissumes "some familiarity with elementary quantum mechanics", as well as a fairly extensive understanding of certain relevant parts of classical theory. Unlike many texts, it begins with the simplest imaginable example of quantum mechanics-photon polarization-and uses it in some detail to illustrate the basic ideas. From here, the book moves at good speed and with commendable clarity through a wide range of standard and not-so-standard topics. In order of appearance, some of the physical applications are: K-mesons, tunnelling, the hydrogen atom, "Cooper pairs", scattering, the Golden Rule, radiation, the Stern-Gerlach experiment, identical particles, atoms, molecules, and relativistic mechanics. Some of the development has clearly been inspired by the Feynman Lectures in Physics (AddisonWesley, 1965).

I enjoyed reading this book, partly because it avoids the standard arrangement of the material to some extent (it must be unusual to find the Aharanov-Bohm effect before the potential barrier in one dimension, and the K-meson before either), and partly because there app zars to be a deliberate attempt to clarify those parts of the development which have elsewhere been glossed over. I particularly liked the good introduction to quantum electrodynamics, and the ten-page derivation of the Dirac equation for the electron ending with the remark that Dirac didn't go to any of this trouble. The usual feeling of something being pulled out of the hat was absent.

I have one major criticism. The author frequently lapses from his usually precise approach to make a remark which is at best unhelpful and at worst wrong. A selection of those I noted follows: an electric intensity is associated with one photon (page 5); the probability amplitude "leaks", "flows", "hops", "diffuses" (47-53); "The $\alpha$ particle will bounce around inside the nucleus" (96); certain elementary particles have "an internal angular momentum, like a ball spinning on its own axis" (302). Statements like these will cause no difficulty for readers who are already familiar with quantum mechanics; on the other hand, the book is meant for readers who are still trying to understand the problems. It is true that an intuitive picture may often produce a comfortable feeling that we have understood what happens, but in some circumstances it may be better to acknowledge that such a picture does not exist.

In short, I recommend this book for its arrangement and mathematical clarity, but caution is necessary at certain points. The printing is clear, though a little untidy in places, and there are very few important misprints.

John L. MaRTin

\section{QUANTUM THEORY PUT SIMPLY}

\section{Elements of Advanced Quantum Theory}

By J. M. Ziman. Pp. xii +269 . (Cambridge University Press: London, July 1969.) 55s; $\$ 9.50$.

This book is unlike any other book on advanced quantum theory that $I$ have seen. Whereas most other books, even if they survey a wide range of topics, go into some matters in detail, this book is unashamedly superficial, and does not even quote the references where more detailed treatments can be found. The object of the book is to familiarize the reader with the language of advanced quantum theory, rather than io teach him how to use the techniques. He should be made aware of the context of the ideas which are used in theoretical paper's, although, without further study and practice, he will not be able to use such ideas himself.

The book consists of two parts. The firse five chapters are concerned mainly with the methods of many-body theory, and the last two are concerned with relativistic formalisms and with symmetry. As one would judge from length alone, the second part is much sketchier than the first. The first part gives a reasonably comprehensive survey of second quantization, perturbation theory, Green functions, and various applications to many-body systems. A few topics have been left out, such as the treatment of superconductivity theory as a generalization of the Hartree-Fock method, and the generalization of ground state approximation methods to problems of thermal equilibrium, presumably because they are too specialized.

In the sixth chapter, classical relativistic field theory is set up and then quantized, spinors are introducad. the Dirac equation is derived, and there is a final discussion of the analytic S-matrix and the Mandelstam representation. No guidance is given to the meaning of Regge poles, bootstraps and the like. The seventh chapter, on tho application of group theory, is more comprehensive, but $I$ think it is too brief to achieve its object. For example, the Wigner-Eckhart theorem is explained in words without being written down. In the final discussion of SU(3) the relation of the strangeness and isotopic spin to the hexagonal pattern of eight particles is not made explicit.

In the first part of the book, and in part of the last chapter, I believe the author has been successful in providing an introduction to the language and ideas of the subject which should bo helpful to students beginning research in experimental and theoretical solid state physics. They should also learn something useful from the discussion of aspects of relativistic problems. The student of elementary particles is likely to find the book less ussful, because he will not be introduced to many of the ideas which are used in research papers of the past few years. Nevertheless, this book is a very welcome addition to the literature. because few people share Ziman's ability to write simply about such topics.

\section{J. THOULESS}

\section{DOPED CHIPS}

\section{Heavily Doped Semiconductors}

By Viktor I. Fistul'. 'Translated from the Russian by Albin Tybulewicz. (Monographs in Semiconductor Physics, Vol. 1.) Pp. xi+418. (Plenum: New York, 1969.) $\$ 25$.

THE tunnel diode was invented in 1958. Its operation depends on the negative resistance of a $\mathrm{p}-\mathbf{n}$ junction made from highly doped $\mathbf{n}$ and $\mathrm{p}$ type semiconductors. 1958 marked a shift of interest, which had previously been largely devoted to lightly doped semiconductors, to include heavily doped semiconductors. Interest in such semiconductors is as great today although it is not confined to their use for tunnel diodes. For example, there is much interest now in the transition from metal to nonmetal, and there is a need for a book or extensive review to collect and criticize all the many aspects of the subject. The original text of Professor Fistul's was published in 1967 but has been revised by the author for this American edition. The author states in the preface of the American edition, however, that the monograph represents the state of knowledge on heavily doped semiconductors 